\title{
Malignant Uterine Corpus PEComa
}

National Cancer Institute

\section{Source}

National Cancer Institute. Malignant Uterine Corpus PEComa. NCI Thesaurus. Code C127072.

A malignant neoplasm with perivascular epithelioid cell differentiation arising from the uterine corpus. The neoplasm is usually a large size, and charcaterized by the presence of marked nuclear atypia, pleomorphism, increased mitotic activity, necrosis, and infiltrative margins. The most common metastatic sites are lungs, lymph nodes, and bone. Patients present with a pelvic mass or abnormal bleeding. 